\title{
ESTUDO DA INTELIGÊNCIA ARTIFICIAL APLICADA NA ÁREA AS SAÚDE
}

\section{STUDY OF ARTICIAL INTELLIGENCE APPLIED IN THE HEALTH AEREA}

\author{
Débora Delfim Guarizi ${ }^{1}$; Eliane Vendramini de Oliveira ${ }^{2}$ \\ ${ }^{1}$ Graduanda em Análise e Desenvolvimento de Sistemas/FATEC-Presidente Prudente; \\ ${ }^{2}$ Docente/ FATEC-Presidente Prudente e FAI-Faculdades Adamantinenses Integradas. \\ e-mail: debora guarizi@hotmail.com
}

RESUMO - O desenvolvimento científico e tecnológico apresenta-se de uma maneira contínua e acelerada, por isso, dentro de diversas áreas da Tecnologia da Informação ( $\mathrm{TI}$ ) a Inteligência Artificial (IA) tem se destacado. A busca pela IA é devido ao seu mecanismo inteligente, sendo possível desenvolver softwares que se podem comportar como um ser humano, sendo assim, um mecanismo inteligente. A IA pode ser aplicada em diversas áreas do cotidiano, um exemplo prático são os programas de diagnostico médico. Com essa diversidade de campos que abrange a $I A$, na área da saúde, surge desenvolvendo equipamentos com o objetivo de realizar diagnósticos médicos. Um pequeno grupo de cientistas da computação e profissionais da saúde dedicou-se a uma nova área de pesquisa, sendo a Inteligência Artificial Médica (IAM). Surgindo os primeiros sistemas desenvolvidos para área da saúde, como: CASNET, MYCIN, PIP.

Palavras-chave: Inteligência Artificial; Inteligência Artificial Médica; Diagnóstico.

ABSTRACT - The scientific and technological development presents a continuous and accelerated way, so within several areas of the Information Technology (IT), Artificial Intelligence (AI) has been highlighted. The search for the Al is due to its intelligent mechanism, it is possible to develop software that can behave as a human being, therefore, an intelligent mechanism. The Al can be applied in many areas of everyday life, a practical example is medical diagnosis programs. With this diversity of fields that Al covers, in health, arises developing equipment for the purpose of performing medical diagnoses. A small group of computer scientists and healthcare professionals devoted to a new area of research, and Medical Artificial Intelligence (MAI). Appearing the first systems developed for healthcare, as CASNET, MYCIN, PIP.

Keywords: Artificial intelligence; Medical Artificial Intelligence; Diagnostic.. 


\section{INTRODUÇÃO}

Um dos grandes desafios da Inteligência Artificial é o desenvolvimento de sistemas que se baseiam nas decisões humanas, estas aplicações são encontradas com frequência na Inteligência Artificial aplicada na Medicina, sendo desenvolvidas a partir de Sistemas Especialistas.

Não é de hoje que a tecnologia da informação é utilizada para estudos de diagnósticos médicos. Robert Ledley e Lee Lusted, descrevem a utilização da lógica simbólica e estatística como métodos para auxiliar no processo de decisão e apontaram o computador sendo 0 instrumento adequado para auxiliar o processo de diagnósticos médicos (Coeira, 2014). Com o encontro da IA e a medicina, ficando conhecida como IAM, sendo uma comunidade de pesquisa, com desenvolvimento de sistemas para o auxilio em diagnósticos. Conforme relata Coeira (Online, 2014), "Inteligência Artificial Médica se preocupa primeiramente com a construção de programas de IA que realizem diagnósticos e fazem recomendações terapêuticas". Os programas de IA em medicina são baseados em modelos simbólicos das entidades nosológicas (classificação das doenças) e suas relações com os fatores ligados ao paciente e às manifestações clínicas. Um dos sistemas mais comuns que são aplicados na área da IAM são os Sistemas Especialistas (SE), estes contém o conhecimento médico, utilizam-se tarefas especificas, capazes de raciocinar a partir dos dados dos pacientes.

Os SE estão inteiramente voltados para o conhecimento simbólico, para desenvolver aplicações com maior semelhança ao comportamento humano. São programas planejados para adquirir e disponibilizar o conhecimento operacional de um especialista humano em uma determinada área ou campo de atuação. Conforme relata Widman (Online, 2014), o $\mathrm{SE}$, é um sistema de suporte a decisão, pois pode efetuar determinadas decisões de duas maneiras: apoio a decisão e tomada de decisão.

Os SE fornecem respostas a determinadas perguntas de uma área especifica, através de inferências humanas, a partir do conhecimento contido em uma base de conhecimento especializado. O sistema deve ter uma capacidade de explicar ao usuário as suas conclusões e o raciocínio para atingir a mesma.

Os softwares aplicados na área da saúde possuem um desenvolvimento inteligente utilizando SE. Não é de hoje que essa aplicação é feita, mas desde quando um dos primeiros sistemas foi desenvolvido, sendo ele o MYCIN.

Conforme Westphal (2003), a evolução da IA nos ambientes médicos, pode 
ser dividida em quatro estágios. A primeira etapa do desenvolvimento da IA na área da saúde inicia-se com o desenvolvimento do sistema CASNET - CAUSAL ASSOCIATIONAL NETWORK (Rede casual associativa) é um tipo de rede semântica, desenvolvido na linguagem Fortran, tendo por objetivo descrever diferentes tipos de processos complexo, mas desenvolvido para descrever fisiopatologias, observações externas por uma estrutura associativa relacionada com estado fisiopatológico. O sistema tinha como por objetivo diagnosticar patologias, como: diabetes, anemias, doenças da tireoide. Mas, acabou-se por auxiliar em diagnostico, prognóstico e terapia do glaucoma.

Sistema também conhecido como, CASNET/glaucoma, baseia-se em uma rede clinica de especialistas em glaucoma, o sistema reconhece a patologia, prescrevendo medicamentos para estados patológicos relacionados ao glaucoma. Utiliza-se estratégias de seleção para um tratamento especifico, acontecendo a partir dos dados individuais de cada paciente para 0 diagnóstico dado. CASNET utiliza uma variedade de esquemas de representação do conhecimento médico em formas modulares e hierárquicas. Desta forma pode-se obter um maior conhecimento sobre como se podem escolher diferentes tipos de métodos de resolução de problemas em diferentes níveis de complexidade para coincidir com a natureza e o grau de dificuldade das tarefas de tomada de decisão. Um dos regimes gerais mais concisas ainda versáteis desenvolvidos até à data é o sistema especialista, que está sendo usada na criação de sistemas de consulta de reumatologia e endocrinologia.

O sistema que marcou na aplicação da Inteligência Artificial na medicina foi o Sistema Especialista MYCIN. Sistema desenvolvido por Edward Shortliffe, onde propôs a construção de um "Especialista Artificial", um conselheiro em antibioticoterapia. Shortliffe desenvolve o sistema especialista como dissertação de doutorado, escrito na linguagem Lisp, tendo como usuários alvo: médicos, paramédicos e estudantes de medicina. Sendo o primeiro programa solucionador de problemas de raciocínio através de informações incertas ou incompletas. Fornecia uma explicação clara e lógica do raciocínio, utilizou-se de uma estrutura de controle apropriado para o domínio do problema específico. É um tipo de Sistema Especialista desenvolvido com Inteligência Artificial especifico para área médica. Projetado para identificar as bactérias causadoras de infecções graves, tais como bacteriemia e meningite, e para recomendar antibiótico especifico, com dose ajustada para o peso corporal do paciente. 0 sistema também foi utilizado para diagnosticar doenças da coagulação 
sanguínea. MYCIN diagnosticaria patologias com base nos sintomas do paciente e testes médicos, caso necessário, o programa solicitaria informações adicionais como exames laboratoriais, chegando a um provável resultado e recomendando-se um tratamento apropriado. Caso necessário MYCIN relatava como atingiu determinado resultado. Projetado para auxiliar no tratamento de meningite, tendo por finalidade tratar infecções graves, selecionando um antibiótico especifico para o paciente com determinada patologia. MYCIN diagnosticaria patologias com base nos sintomas do paciente e testes médicos, caso necessário, o programa solicitaria informações adicionais como exames laboratoriais, chegando a um provável resultado e recomendaria um tratamento apropriado. Caso necessário o MYCIN relatava como atingiu determinado resultado. O programa utiliza o tipo de raciocínio backward chaining, encadeamento para trás ou raciocínio para trás. Esse raciocínio refere-se a um método de inferência utilizado em IA, onde tem-se por objetivo trabalhar para trás para descobrir como atingir o objetivo final. Para que isso aconteça é necessário que a IA trabalhe com um sistema baseado em regras.

Conforme Westphal (2003), desenvolvimento do PIP Presente IIIness Program (Programa de doença Atual), finalizou a primeira etapa da IA na medicina. Sendo uma ferramenta de diagnóstico precoce projetado para emular os médicos na avaliação de pacientes com edema, utilização em consultas e diagnósticos na medicina interna e na nefrologia (de acordo com o Dicionário Aurélio, Nefrologia é a especialidade médica que se ocupa do diagnóstico e tratamento clínico das doenças do sistema urinário, em especial o rim). O sistema auxiliava os especialistas humanos através de fatos sobre o paciente a partir de um banco de dados desenvolvendo uma hipótese sobre o que o paciente sentia. O sistema tem quatro componentes principais: conjunto de dados do paciente, memória de longo prazo, repositório de conhecimento, memória de curto prazo, cruzamento de dados do paciente e do repositório de conhecimento, um programa de supervisor para facilitar conhecimento e incorporar as contribuições do paciente.

O funcionamento do sistema se dá a partir dos dados clínicos do paciente inseridos por um especialista humano. O programa supervisor verifica os dados relevantes para a memória de curto prazo e definindo-as como fatos ativos ou não para o diagnóstico do paciente, conteúdo armazenado no banco de dados. Fatos que o sistema apenas verificou e não foi selecionado como ativos para o diagnósticos do paciente ficam marcados como semi- 
ativos. Consequentemente o sistema acabaria por agregar fatos obtidos no banco de dados e conselhos do especialista humano, o sistema em questão, sugere mais perguntas para ajudar o clinico a focar na patologia em questão, sugerindo ao usuário, validar os dados hipotéticos. As hipóteses são geradas por analisar todos os fatos em questão, fatos estes que são encontrados no repositório de conhecimento. Os fatos são conjunto de regras que permite que 0 sistema determine se é ou não uma afirmação valida para o paciente em questão. O programa PIP não está em uso comercial elevado, mas algumas funções do sistema pode-se ser encontrada nos sistemas clínicos atuais. O sistema pode ser visto como um dos sistemas que auxiliam no desenvolvimento da base de sistema de apoio a decisão. A segunda etapa do desenvolvimento da IA na medicina ficou marcada pelo surgimento dos seguintes sistemas: EMYCIN, Expert e Age. Os sistemas foram desenvolvidos para aplicação em controle de instrumentos de UTI, interpretação de teste laboratorial para aconselhamento de diagnóstico e tratamento de doenças. Neste período houve o desenvolvimento e disseminação das ideias dos Sistemas Especialistas, de shells para representar o conhecimento e das decisões baseadas em heurísticas. (1995 apud, BRONZINO; WESTPHAL, 2003).
O principal objetivo dos sistemas Shells é separar a base de conhecimento da máquina de inferência. Assim, o usuário do sistema deve preocupar-se em apenas obter conhecimento do especialista humano, pois a máquina de inferência é inerente ao sistema. O sistema Expert System Shell foi desenvolvido com o objetivo de desenvolver o ETs - Expertise Transfer System (Sistema de Transferência de Conhecimento) específico. São Sistemas Especialistas incompletos, que requerem um menor esforço, a fim de mobilizar um sistema real. Auxiliam a construção de Sistemas Especialistas para diagnóstico médico, originou-se do CASNET.

De acordo com Bundy e Wallen (Online, 2014) Emycin é um Sistema Especialista shell implementado em Interlisp, sendo uma versão dependente do Mycin. Criado para auxiliar sistemas e utiliza um encadeamento para trás, assim sendo uma estratégia de controle. Desenvolvido sem uma base de conhecimento e reutilizando a interface do usuário e o motor de inferência para que o sistema não fosse construído a partir do zero. Portanto, sendo de melhor utilidade para profissionais não especialistas. O objetivo geral do projeto $A G E$ Attempt to Generalize (Tentativa de Generalizar), é desmistificar para tornar explicita a engenharia do conhecimento. Conforme relata Nii e Aiello (Online, 1979) o objetivo geral do projeto AGE - Attempt to 
Generalize (Tentativa de Generalizar), é desmistificar para tornar explicita a engenharia do conhecimento. A concepção e implementação do programa baseia-se na construção dos softwares, desenvolvendo programas com mais facilidade, a partir de sistemas já implementados com uma base de conhecimento e facilitando as técnicas de IA que serão aplicadas não havendo a necessidade de reprogramar um sistema e cada problema específico, auxiliando especialistas humanos com menos conhecimento. A única restrição para um especialista da área da computação desenvolver sistemas a partir do AGE é ter conhecimento na linguagem, pois o sistema é implementado em Interlisp. Os componentes básicos do sistema já estão programados, isso se da pelo fato do sistema estar escrito em módulos, possibilitando o usuário modificar sua implementação. As vantagens para a utilização do Age são: os componentes básicos do sistema já estão programados, AGE permite que o usuário utilize diferentes resoluções de determinado problema.

De acordo com Westphal (2003) o terceiro estágio da IA na medicina período que marca a iniciativa de pesquisadores de IA no desenvolvimento de formas representativas para o raciocínio médico. Iniciando-se os conceitos de Redes Neurais Artificiais (RNA) para a aplicação na informática médica. Assim, existiu um interesse na área de RNA e nas estruturas conexionistas, aplicando-se um aprendizado de tomada de decisões desenvolvendo-se o método backpropagation. O neurônio artificial, é uma estrutura lógica-matemática que procura simular a forma 0 comportamento e as funções de um neurônio biológico, baseando-se na captação de processamentos e memória das informações humana, sendo estas máquinas, baseadas na arquitetura do cérebro humano. As redes neurais artificiais é uma forma de sistema computacional desenvolvidos com vários processadores, com elementos de processamento simples, mas com um grau elevado de interligações. Um neurônio biológico pode ter até 10.000 entradas diferentes, e pode enviar a sua saída (a presença ou ausência de um pico de curta duração) para muitos outros neurônios. Os neurônios são conectados em um teste padrão 3-dimensional. Os cérebros reais, no entanto, são ordens de magnitude mais complexa do que qualquer rede neural artificial até agora considerada. Combinando diversos neurônios pode-se formar o que chamam de rede neural artificial.

Existem diversas variantes para uma rede neural, e suas combinações, podem-se modificar a arquitetura da aplicação conforme sua necessidade, ou até mesmo, conforme o desejo do projetista. Por tanto, os itens que compõe uma rede neural, com 
possibilidades a modificações, são: conexões entre camadas, camada intermediaria, quantidade de neurônios, função de transparência, algoritmo de aprendizado.

As RNAs podem ser aplicadas na área médica sendo conhecidas como Computação Bioinspirada. Esta é a área de pesquisa da computação que estuda e desenvolve técnicas computacionais inspiradas na biologia resolvendo problemas práticos. Problemas este como o DNA, para obter-se os resultados de um material genético, o computador agrupa dados biológicos e os classifica-os. Além dos testes poderem fazer o reconhecimento de paternidade, pode-se realizar comparação entre espécies de animais.

Outra aplicação na medicina é o descobrimento de determinadas patologias através do DNA, podendo até mesmo descobrir as chances que o paciente desenvolver determinada doença e os tipos de problemas.

Encontra-se a utilização das redes neurais artificiais nos Sistemas de Apoio a Decisão Clinica. Softwares desenvolvidos para aprenderem com base nos dados inseridos e ajudarem na analise de dados como imagens de mamografias, colonoscopias ou mesmo dados de acompanhamento clinico para prognósticos.

O quarto estágio da Inteligência Artificial na saúde é o contínuo desenvolvimento de representações baseadas em conhecimento, uso de raciocínio qualitativo, modelagem e controle de processos críticos, métodos estatísticos, redes Bayseanas nos processos de tomada de decisão, ratificação de uso de redes neurais.

Para o desenvolvimento da IA na área médica é necessário a utilização dos SE, sendo desenvolvidos baseados no conhecimento de um especialista humano. Sistemas estes capazes de analisar e diagnosticar determinada patologia em analise, simulando o raciocínio e conhecimento de um médico.

Os SE especialistas desenvolvidos para área médica são sistemas de tomada de decisão. As tomadas de decisões ocorrem em diversas situações nas atividades medicas. Existindo três importantes situações nas quais os computadores podem auxiliar na tomada de decisão, sendo relacionadas à medicina clinica, sendo: diagnósticos, prognósticos, planejamento terapêutico.

A utilização dos SE na cardiologia se dá por uma análise de dados hemodinâmicos (de acordo com o Dicionário Aurélio hemodinâmico é o termo da fisiologia referente ao estudo de fenômenos que fazem menção a circulação sanguínea) obtidos por um Cateter de Swan-Ganz (é um cateter flexível e fabricado em poliuretano que, introduzido através de uma veia central de adequado calibre, chega às estruturas 
cardíacas e pulmonares. É inserido para obter dados muito precisos e indicado na terapia para o controle do estado hemodinâmico do paciente crítico e, sobretudo, se está em estado de choque) a utilização do software é de maior eficaz do que um especialista humano, pois o SE verifica com uma melhor conduta um paciente com choque cardiogênico. A Figura 27 representa uma SE sendo utilizado para analise de uma paciente, verificando se este está ou não em estado hemodinâmico.

Um SE na medicina, Quick Medical Reference - QMR (Sistema de Referência Médica Rápida), desenvolvido para auxiliar em diagnósticos de patologias em pacientes adultos, fornecendo acesso a informações de doenças mais comuns, incluindo históricos médico, sintomas, sinais físicos e os resultados dos testes laboratoriais. Sendo uma ferramenta de diagnostico clínico, que consiste em descrições de mais de 750 patologias.

Um dos maiores desafios tecnológicos, sendo desenvolvido com uma enorme ambição, é o desenvolvimento de sistemas computadorizados utilizando a Inteligência Artificial. Desenvolvimento estes que relacionam médicos e cientistas há um bom tempo, esta tecnologia é considerada de grande potencial na medicina. Com 0 advento de computadores hábeis a armazenar e processar vasta quantidade de informações surgia à possibilidade de que se tornassem verdadeiros "médicos em caixas", auxiliando e ultrapassando os médicos. Para que os desenvolvimentos de sistemas utilizando a Inteligência Artificial iniciassem, um grupo de cientistas e profissionais da área da saúde inicializou um programa de pesquisa para uma nova disciplina chamada Inteligência artificial na Medicina, sendo um Sistema de Apoio à Decisão Médica - SADM. O SADM é baseado em princípios de analises de decisões, que organiza e esclarece informações importantes quanto aos riscos e benefícios de cada tratamento possível, simplificando o processo de decisão para o paciente e para o medico. Mas, apesar desta ajuda, o paciente ainda precisa fazer a sua opção de tratamento e confiar no medico que o acompanha para que este possa explicar com maiores detalhes, os riscos de cada procedimento. Este método de analise que possui vantagens e desvantagens está sendo cada vez mais usado para fornecer as opções mais simples dentro da enorme variedade de alternativas avaliadas. Os Sistemas de Apoio a Decisão Médica podem ser classificados da seguinte maneira: Sistemas com capacidade de decisão própria limitada ou ausente (recuperação de dados sobre paciente, cálculos matemáticos assistidos por computador, análise e interpretação primária de dados) e Sistemas com capacidade de raciocínio automático e 
de inferência (sistemas de classificação de doenças, sistemas especialistas baseados em consulta, sistemas especialistas baseados em criticas). SADM possui os seguintes módulos: perfil do paciente, diagnóstico, avaliação do paciente, análises.

Atualmente existe uma resistência aos usuários, especialistas humanos, a utilizarem sistemas médicos, além de uma série de razões para que os profissionais ainda não utilizem maciçamente os sistemas de apoio à decisão, sendo algum dos motivos: medo da perda da ligação com o paciente, medo da perda do controle da situação, inércia, não aceitação da capacidade da maquina, suspeita na Inteligência Artificial, descontentamento com a entrada de dados, crença de que há uma idade para se aprender a usar o computador.

Um das aplicações do SADM é o sistema DXplain, onde pode auxiliar no diagnostico através da analise de achados clínicos como sinais, sintoma e dados laboratoriais, produzindo uma lista dos diagnósticos mais prováveis. O sistema justifica cada diagnostico, quando necessário sugere exames adicionais. A base de dados do DXplain contem cerca de 4,5 mil sintomas associados aproximadamente a 2 mil doenças. A característica importante deste sistema é o poder de relacionar os sintomas e apresentar as referencias bibliográficas em que estão fundamentados. O sistema é utilizado em grande número de hospitais e escolas médicas dos EUA - Estados Unidos da América.

Outro sistema que é dado como exemplo de SADM é o software Risco Cirúrgico MED. Após a inserção dos dados clínicos, ele avalia o risco cardiológico e pulmonar do paciente, facilitando a avaliação pré-operatória para o clínico ou cardiologista. Inteligência Artificial foi além da ficção científica, hoje, é um amplo campo de estudo e em constante mudança. No caso da medicina, as suas aplicações são extensas.

$\mathrm{Na}$ medicina, o diagnóstico e o tratamento de doenças é um ramo que tem sido extensivamente estudada e com a ajuda da robótica têm alcançado grandes resultados que ajudam no cuidado e tratamento de pacientes. A primeira vez que foi utilizado um robô na Medicina foi em 1985, sendo conhecido como PUMA 560, este mecanismo foi desenvolvido para ser utilizado durante a realização de uma biopsia no cérebro para guiar uma agulha.

Um dos problemas, da medicina, que preocupa muito os profissionais e pacientes são as graves infecções hospitalares, manter a limpeza dos locais que tem grande chance de infecção é extremo desafio. Pensando neste problema, pesquisadores desenvolveram o Xenex, Sistema de Desinfecção UV. O dispositivo libera ao meio, ondas que são chamadas de "UV de 
xenônio", um tipo de luz ultravioleta que esteriliza e mata organismos microbiológicos que possa transmitir qualquer tipo de contaminação.

O hospital Albert Einstein adquiriu a tecnologia robótica em 2008, desde lá, equipe de médico especialistas em cirurgias robóticas vem utilizando o Vinci Surgical System, um robô cirurgião desenvolvido pela Intuitive Surgical, empresa que lidera tecnologia de robótica aplicada em cirurgias.

\section{METODOLOGIA}

Por ter o propósito de explorar trabalhos já abordados, especificando detalhes da aplicação da IA e assim abrangendo técnicas da IA aplicada na área da saúde, foi utilizado um método dedutivo.

Compreendendo-se os fenômenos a partir da perspectiva de especialistas, a forma de abordagem do trabalho foi qualitativa.

O objetivo da pesquisa é exploratória, pois utiliza-se do levantamento bibliográfico e aplicações já efetuadas na área.

A natureza da pesquisa é pura ou básica, cujo objetivo é relatar as informações existentes sobre o tema abordado, atendendo à necessidade de obter conhecimento a respeito.

Os procedimentos técnicos utilizados foram: pesquisa bibliográfica, os materiais da qual foram obtidas as informações são de livros e documentos científicos.

\section{RESULTADOS}

Dentre todos os desenvolvimentos que foram pesquisados a partir de uma análise exploratória sobre a Inteligência Artificial aplicada na área da saúde, analisase que, são de extrema importância para a medicina. Pois, os mesmos, relatam o diagnóstico de um determinado paciente, a partir de uma base de conhecimento contida em uma base de conhecimento especializado, havendo uma grande eficácia no resultado das patologias. Pode-se estes sistemas auxiliarem especialistas humanos e até mesmo especialistas com menos conhecimento na área da saúde. Portanto, o sistema deve ter uma capacidade de explicar ao usuário as suas conclusões e o raciocínio para atingir tal resultado.

\section{DISCUSSÃO}

Os sistemas que são desenvolvidos com IA utilizando-se uma base de conhecimento podem auxiliar especialistas humanos em relatar tipos de patologias e até mesmo determinados diagnósticos. Mas, alguns sistemas com seu grau de decisão elevado, nas áreas especialistas da medicina, podem verificar com uma melhor conduta o estado de uma paciente sem que haja a necessidade do programa solicitar 
informações adicionais e até mesmo relatando como chegou a determinado diagnóstico. Isso acontece devido aos Sistemas Especialistas facilitarem a solução de problemas de um determinado campo de aplicação, fazendo inferências a partir de uma base de conhecimento desenvolvido por um especialista humano.

Uma das grandes discussões é se a máquina irá substituir homem na área médica. Desde a Revolução Industrial a máquina vem tomando forças e substituindo o homem na indústria, principalmente em serviços pesados. Essa substituição ficou cada vez mais comum e de certa forma "obrigatória", este fato obteve-se pelos motivos das empresas manterem seus lucros competitivos no mercado.

Já na medicina, existe o grande dilema, as cirurgias assistidas substituíram o especialista humano?

As cirurgias robóticas já enfrentaram preconceitos por parte de médicos e pacientes, esse tipo de preconceito está se tornando mais comum, tanto para pacientes quanto cirurgiões. Pois, estão analisando os benefícios da cirurgia minimamente invasiva.

\section{CONCLUSÃO}

Tendo como metodologia para o desenvolvimento deste trabalho uma pesquisa exploratória chega-se a resultados satisfatórios, onde pode-se, concluir que o uso da IA sendo desenvolvida com base de conhecimento de Sistemas Especialistas tem grande influência em diagnósticos para determinadas patologias. Podendo o sistema a partir de um estado especifico de um paciente analisar o seu estado e diagnosticálo.

Por tanto conclui-se que os sistemas desenvolvidos com Inteligência Artificial para a área da saúde podem em determinados casos substituir um especialista humano devido a sua base de conhecimento e suas tomadas de decisão. Para que um SE substitua um sistema especialista humano o software terá que possuir um elevado grau de especificação, assim tendo um melhor desempenho.

Mas, analisando-se a cirurgia robótica deveria ser pensada como cirurgia assistida por robôs. Afinal, é o cirurgião que realiza a cirurgia através de extensores mecânicos, que tem como principal função proporcionar mais destreza a operação. A máquina ainda não substituiu o homem na medica, pois ainda necessita de um especialista humano para intervir nas cirurgias, diagnósticos.

\section{REFERÊNCIAS}

BUNDY, A.; WALLEN, L. Emycin. Disponível em:

<http://link.springer.com/chapter/10.1007\% 2F978-3-642-96868-6_66>. Acessado em: 15 maio 2014.

COIERA, E. W. Inteligência artificial na Medicina. Disponível em: 
http://www.informaticamedica.org.br/infor maticamedica/n0104/coiera.htm>. Acessado em: 21 abr. 2014.

NII, H. P., AIELLO, N.; AGE (Attempt to Generalize): A Knowleg - Based Program for building Knowledge - Based Programs. Disponível em: <https://saltworks.stanford.edu/catalog/drui d:qp661zp6387>. Acessado em: 11 Maio 2014.

STAIR, R. M. Sistemas de informação: uma Abordagem Gerencial. 2Ed. Rio de Janeiro: LTC, 1998, p. 260.

SZOLOVITS, P.; PAUKER, S.G. Categorial and probabilistic reasoning in medical diagnosis, Artificial Intelligence. Vol. 11, p. 115 - 144, 1978. http://dx.doi.org/10.1016/00043702(78)90014-0

WESTPHAL, J. T.; Modelagem Difusa de um Sistema Especialista Médico: Avaliação dos Fatores de Internação em Crianças Queimadas. 2003. 123f. Dissertação (Mestrado) - Programa de Pós - Graduação em Ciência da Computação, Universidade federal de Santa Catarina, Florianópolis, 2003.

WIDMAN, L. E. Sistemas Especialistas em Medicina. Disponível em: http://www.informaticamedica.org.br/infor maticamedica/n0105/widman.htm>. Acessado em: 11 Maio 2014. 